UVX 2008 (2009) 101-106

(C) EDP Sciences, 2009

DOI: $10.1051 / \mathrm{uvx} / 2009016$

\title{
Poudres et couches minces fluorescentes préparées par sol-gel : un exemple de luminophores nanostructurés fonctionnels
}

\author{
R. Mahiou, G. Chadeyron, D. Boyer, P. Boutinaud, \\ A. Potdevin et D. Zambon
}

\author{
Laboratoire des Matériaux Inorganiques UMR 6002 CNRS, Université Blaise Pascal, \\ 24 avenue des Landais, 63177 Aubière Cedex, France
}

\begin{abstract}
Résumé. La nécessité de développer de nouveaux dispositifs de visualisation, d'affichage, d'éclairage ou susceptibles de convertir efficacement l'énergie solaire, à la fois performants, respectueux de l'environnement et possédant un encombrement réduit a permis de relancer fortement l'industrie des matériaux fluorescents à vocation de luminophores. Un des challenges à relever réside dans la réalisation de matériaux à la "demande" possédant, en plus des caractéristiques physico-chimiques, une versatilité de forme et de morphologie. Même si une recherche intense reste toujours consacrée au développement de nouvelles familles de matériaux compatibles avec l'une des applications visées, la tendance actuelle est toutefois fortement orientée vers l'amélioration des propriétés émissives des luminophores déjà existants. Appliqué aux matériaux inorganiques ou aux matériaux hybrides organiques-inorganiques, activés par des ions de terres rares, le procédé sol-gel permet à la fois d'obtenir des poudres à granulométrie contrôlée et des films de qualité en utilisant des techniques de dépôt comme le spray ou le dip-coating. Tout en conservant des caractéristiques structurales et des propriétés spectrales intactes, les matériaux solgel présentent souvent des propriétés améliorées par rapport à leurs homologues issus de techniques de préparation classiques comme la céramisation, malgré des températures de préparation plus basses. On peut attribuer ces spécificités à la méthode de synthèse elle-même qui permet de structurer les matériaux en utilisant des mécanismes réactionnels à l'échelle moléculaire, conduisant à des matériaux plus homogènes et plus purs. Quelques exemples de composés sont présentés pour illustrer l'utilisation de cette technique pour préparer de façon originale des matériaux excitables sous UV-VUV ou sous RX.
\end{abstract}

\section{INTRODUCTION}

Si les synthèses dites classiques sont encore largement utilisées pour préparer des luminophores ou des scintillateurs, un effort important a été consacré ces dernières années au développement de nouvelles voies de synthèse, notamment le procédé sol-gel. Ce procédé est rapidement apparu comme une technique autorisant l'élaboration et la mise forme, de manière aisée, de matériaux nanostructurés aux propriétés souvent exaltées. Plusieurs familles de verres, de céramiques ou d'hybrides peuvent ainsi être élaborées à partir d'alcoxydes judicieusement choisis [1-3].

Parmi les diverses compositions élaborées, nous retiendrons les borates comme $\mathrm{LnBO}_{3}$ [4] et $\mathrm{Ln}_{3} \mathrm{BO}_{6}$ [5], les aluminates comme $\mathrm{Ln}_{3} \mathrm{Al}_{5} \mathrm{O}_{12}$ [6], ou les phosphates $\mathrm{LnPO}_{4}$ ou $\mathrm{LnP}_{4} \mathrm{O}_{12}$ [7] $(\mathrm{Ln}=\mathrm{Lu}$ ou Y, Gd) activés par $\mathrm{Eu}^{3+}$ ou $\mathrm{Tb}^{3+}$ voire $\mathrm{Ce}^{3+}$, le titanate $\mathrm{CaTiO}_{3}: \mathrm{Pr}^{3+}$ [8] ou les silicates $\mathrm{Ln}_{2} \mathrm{SiO}_{5}$ [9] $(\mathrm{Ln}=\mathrm{Y}, \mathrm{Lu})$ dopés par $\mathrm{Ce}^{3+}, \mathrm{Tb}^{3+}$ ou $\mathrm{Eu}^{3+}$. De même, des fluorures $\mathrm{NaLnF}_{4}, \mathrm{LiLnF}_{4}$ [10] et des matériaux hybrides organiques-inorganiques de classe II (de type silice, chromophores) [11] ont été élaborés en utilisant la technique sol-gel. En plus d'être très luminescents sous des excitations UV ou VUV, ces matériaux présentent des potentialités d'application qui paraissent plus pointues et mieux adaptées au domaine de l'éclairage ou à celui de la scintillation. Une maîtrise des procédés alliée à des techniques de mise en forme ou à de nouvelles voies originales de préparation permettent en effet des reproductibilités et des gains en performance tout à fait spectaculaires. Deux exemples d'études (matrices YAG et $\mathrm{LnBO}_{3}$ ) seront présentés dans ce qui suit. 


\section{2. ÉLABORATION DES POUDRES ET DES COUCHES MINCES DES MATÉRIAUX INORGANIQUES}

Les précurseurs utilisés sont généralement des alcoxydes simples. Pour $\mathrm{YBO}_{3}$ dopé par l'ion $\mathrm{Eu}^{3+}$ par exemple, la premire étape consiste à synthétiser les alcoxydes d'yttrium et d'europium à partir de chlorures d'yttrium et d'europium anhydres. Un isopropoxyde de potassium est ajouté à la solution précédente et conduit à l'élimination des chlorures par précipitation de $\mathrm{KCl}$. Finalement, des quantités déterminées de triisopropoxyde de bore et d'acétylacétone (acacH) sont ajoutées au milieu réactionnel. L'ajout d'acacH diminue fortement le risque d'hydrolyse et conduit à l'obtention d'un sol stable et homogène. En fin de synthèse, le mélange est centrifugé afin d'éliminer le $\mathrm{KCl}$ et le sol est obtenu. Suivant l'application souhaitée, plusieurs solutions sont ensuite envisageables : élaboration de poudres ou de revêtements.

Ce protocole a été adapté pour chacune des formulations citées précédemment. Les profils de traitements thermiques sont définis précisément de façon à conduire à la densification du matériau et au contrôle de son état de cristallisation. Cette étape est cruciale, car elle conditionne la coalescence des grains par frittage et la cristallisation des couches, ce qui peut s'avérer néfaste par exemple pour l'utilisation en configuration de guidage optique. Dans le cas de l'application à la réalisation d'écrans scintillants sous rayonnement $X$, pour lesquels l'efficacité est conditionnée entre autres par l'épaisseur, nous avons mis au point une technique de dépôt par spray permettant l'obtention de couches épaisses (quelques $\mu \mathrm{m}$ ). Ce type de couches ne possède pas une qualité permettant un guidage optique, mais présente l'épaisseur et la compacité compatibles avec une application en scintillation.

Les propriétés physico-chimiques des différents matériaux élaborés sont caractérisées par des techniques de détermination de l'ordre moyen, comme la diffraction des rayons $\mathrm{X}$, le plus souvent associées aux spectroscopies de l'ordre local comme la RMN, la RPE, la diffusion Raman, l'absorption infrarouge et l'absorption X. Ces caractérisations sont complétées suivant le cas par des profils de composition par XPS ou RBS et par des études optiques (ellipsométrie, profilométrie, fluorescence et scintillation). Une attention particulière est accordée à la caractérisation morphologique et texturale des matériaux en faisant usage des microscopies MEB, MET et MFA ainsi que la granulométrie par diffusion laser.

\section{LUMINOPHORES PRÉPARÉS PAR VOIE SOL-GEL : POUDRES ET REVÊTEMENTS POUR L'ÉCLAIRAGE, LA VISUALISATION, OU LA SCINTILLATION}

\subsection{Vers la synthèse et l'élaboration orientées}

Il nous a semblé important d'insister, notamment pour les matériaux sol-gel, sur l'importance que revêt la connaissance des propriétés de l'état sol. Pour ce faire, nous présenterons quelques résultats relatifs à la matrice $\mathrm{YAG}\left(\mathrm{Y}_{3} \mathrm{Al}_{5} \mathrm{O}_{12}\right)$ tout en sachant que ce type d'étude est étendu aux autres compositions. L'étape cruciale lors de l'élaboration des matériaux par le procédé sol gel correspond au passage par un état sol qui peut être suivant l'application finale souhaitée (poudre ou films minces) stabilisé ou non. L'ajout d'un agent chélatant comme l'acétylacétone $(\mathrm{acacH})$ lors de la synthèse de la matrice YAG a permis d'obtenir un sol stable et homogène. L'existence d'un taux optimal d'acacH au delà et en deçà duquel le rôle de cet agent est inefficace a été mis en évidence par spectroscopie UV-Visible (suivi de cinétique d'hydrolyse à $800 \mathrm{~nm}$ ), comme l'illustre la figure 1 . La zêtamétrie a permis de confirmer ces résultats. Ces études sur l'état sol et sur son évolution après hydrolyse se sont poursuivies par des mesures SAXS qui ont permis d'accéder à la structuration du réseau inorganique. Cette étude réalisée sur la ligne XRD2 au Brazilian Synchrotron Light Laboratory (LNLS) à Sao Paulo a été menée en collaboration avec Celso Santilli (Instituto de Quimica, Araraquara (Brésil) et Valérie Briois (ligne SAMBA@SOLEIL). Le rôle de l'acacH dans la structuration du sol de YAG a clairement été mis en évidence par l'enregistrement des courbes SAXS et XAS comme l'illustrent les figures 2 et 3. 


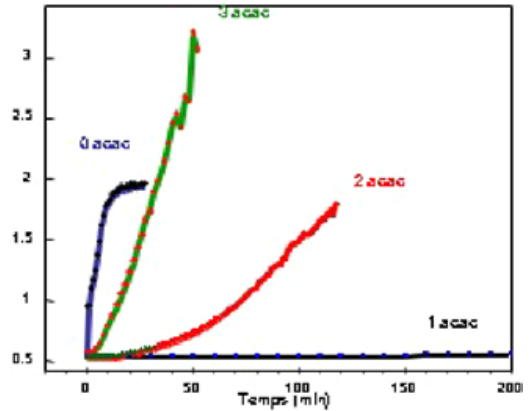

Figure 1. Évolution de l'absorbance à $800 \mathrm{~nm}$ après hydrolyse $60^{\circ} \mathrm{C}$.

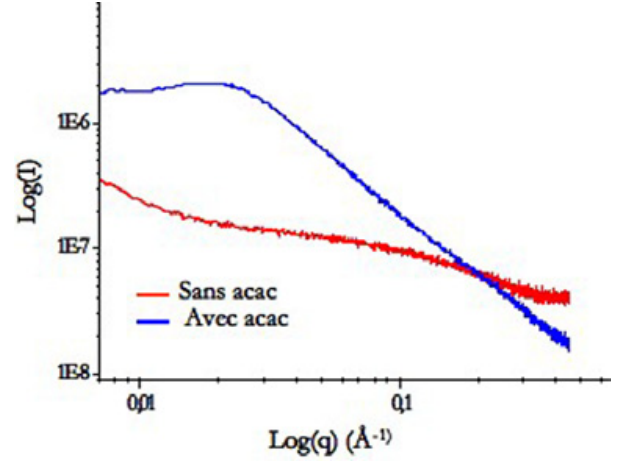

Figure 2. Courbes de diffusion des rayons $\mathrm{X}$ réalisées sur des sols de YAG : $20 \% \mathrm{~Tb}^{3+}$.

L'analyse des courbes SAXS a permis de montrer que le sol non stabilisé est constitué de particules primaires ayant des dimensions de l'ordre du nanomètre. Le sol stabilisé avec une quantité d'acacH optimisée présente quant à lui une dimension fractale (D) indiquant que nous sommes en présence de fractal de masse. La taille mesurée des agrégats fractals se situe au voisinage de $9 \mathrm{~nm}$ (Table 1).

Tableau 1. Valeurs du rayon de giration Rg et de l'indice de dimension fractale D des sols.

\begin{tabular}{lll}
\hline Echantillon & $\operatorname{Rg}(\mathrm{nm})$ & $\mathrm{a}($ pente $)$ \\
\hline Sol non stabilisé & 0,9 & \\
Sol stabilisé & $8,9 \quad-1,5$ \\
\hline $1<|\mathrm{a}|<3:$ fractale de masse $|\mathrm{a}|=\mathrm{D}$ \\
$3<|\mathrm{a}|<4:$ fractale de surface $\mathrm{D}=6-|\mathrm{a}|$
\end{tabular}

Parallèlement, l'étude XAS réalisée sur ces mêmes sols au seuil K de l'yttrium a permis de mettre en évidence une organisation déjà avancée pour le sol de YAG stabilisé contrairement au sol non stabilisé (figure 3). Les résultats issus de l'analyse des spectres EXAFS enregistrés pour le sol stabilisé et le sol non stabilisé sont regroupés dans la Table 2.

Nous avons montré que l'environnement de l'atome d'yttrium dans le sol stabilisé est constitué de 8 atomes d'oxygènes, 4 à une distance de $2,30 \AA$ et 4 à une distance de 2,43 $\AA$. Les distances Y-O ainsi obtenues sont en parfait accord avec les valeurs proposées lors de la description structurale de la matrice YAG [12], ce qui n'est pas le cas pour le sol non stabilisé. De plus, l'affinement du second pic, seulement possible pour le sol stabilisé, a permis de montrer que la seconde sphère de coordination de $\mathrm{l}^{\prime}$ atome d'yttrium contient des atomes d'aluminium. Le sol stabilisé contient donc des alcoxydes doubles (Y-Al) et non uniquement des alcoxydes simples comme il était possible de l'envisager. Le sol stabilisé est beaucoup mieux organisé que son homologue non stabilisé et cette structuration précoce est proche de celle du matériau final.

La stabilisation du sol par l'acétylacétone a des répercutions sur la morphologie du matériau final (poudre traitée $4 \mathrm{~h}$ à $1100^{\circ} \mathrm{C}$ ) comme l'illustrent les clichés de MEB présentés sur la figure 5. L'image associée à l'échantillon stabilisé se caractérise par des grains de très petites tailles et par une faible porosité comparativement au composé non stabilisé. L'étude de la cinétique de gélification des sols en fonction de l'ajout ou non d'agent chélatant et de l'âge des sols se poursuit en collaboration avec Valérie Briois et Celso Santilli. Les premiers résultats XAS et SAXS relatifs à cette problématique ont pu clairement mettre en évidence une évolution des sols dans le temps (suivi des sols sur une période de 18 mois). Le vieillissement des sols, qui n'avait pas été pris en compte jusqu'ici, s'avère être un paramètre non négligeable ayant des implications sur les propriétés optiques du matériau final. Un temps 


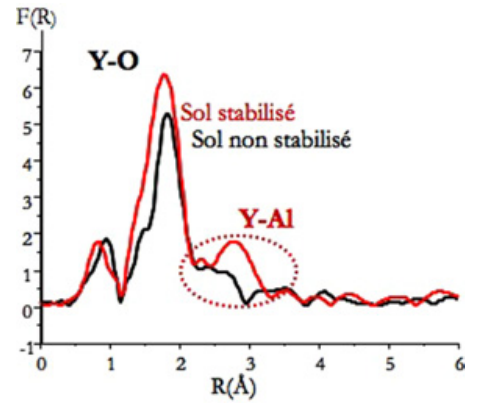

Figure 3. Pseudo-modules des transformées de Fourier obtenues au seuil $\mathrm{K}$ de l'yttrium dans les sols de YAG : $20 \% \mathrm{~Tb}$.
Tableau 2. Paramètres structuraux des sols déduits de l'affinement EXAFS présenté ci-contre et comparés à ceux de la référence YAG [12]. N, d et $\sigma$ représentent le nombre de coordination, la distance et le facteur de Debye-Waller respectivement.

\begin{tabular}{clclc}
\hline Echantillon & Atome & $\mathrm{N}$ & $\mathrm{d}(\AA)$ & $\sigma(\AA)$ \\
\hline Sol non stabilisé & $\mathrm{O}$ & 1,1 & $2,33(2,30)$ & 0,12 \\
& $\mathrm{O}$ & 7,3 & $2,43(2,43)$ & 0,027 \\
\hline Sol stabilisé & $\mathrm{O}$ & $4,6(4)$ & $2,30(2,30)$ & 0,062 \\
\cline { 2 - 4 } & $\mathrm{O}$ & $3,7(4)$ & $2,43(2,43)$ & 0,068 \\
& $\mathrm{Al}$ & $2,2(2)$ & $2,95(3,00)$ & 0,1 \\
& $\mathrm{Al}$ & $1,1(4)$ & $3,30(3,35)$ & 0,064 \\
\hline
\end{tabular}

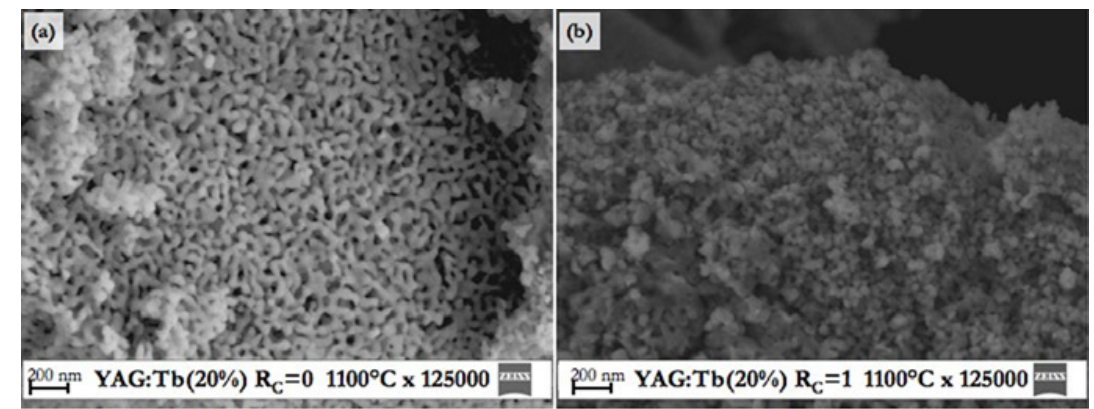

Figure 4. Clichés MEB de YAG $: \mathrm{Tb}^{3+}\left(1100^{\circ} \mathrm{C}\right)$ a) non stabilisé, b) stabilisé avec acacH $(\mathrm{acacH} / \mathrm{Al}=1)$.

de mûrissement optimal des sols a pu être mis en évidence à partir de l'étude optique. L'enregistrement des spectres XAS a montré que l'organisation des sols (en terme de structuration) évolue de façon croissante depuis son élaboration jusqu'à un temps optimal. Au-delà de cette valeur le sol commence à se désorganiser et cette désorganisation s'accentue avec le temps. L'enregistrement des spectres d'émission sous excitation bleue a montré que les poudres calcinées à $1100^{\circ} \mathrm{C}$, issues de sols d'âges et maturités différents, suivaient la même évolution.

\subsection{Vers le développement de sources d'éclairage propres et économes}

La réalisation de panneaux ou de murs de lumière pour l'éclairage ou la décoration est un secteur en plein essor. Deux axes de développement sont actuellement retenus, l'un basé sur l'électroluminescence et l'autre sur la photoluminescence obtenue sous irradiation UV-bleue ou VUV. Nous avons développé une forte expertise dans le domaine de la réalisation de films sol-gel luminescents. Diverses techniques de dépôts (dip et spin coating, spray) ont permis de préparer des films homogènes de qualité, opaques et épais (plusieurs microns), ainsi que des films minces transparents ( $20 \mathrm{~nm}$ à $2 \mu$ m environ). Le challenge à relever est de réaliser des couches transparentes suffisamment épaisses ou structurées en puits quantiques pour que le guidage induit par la faible épaisseur (à l'origine de la perte de plus de $80 \%$ de la lumière émise à travers les tranches) soit limité. Nous avons récemment développé plusieurs familles d'oxydes en films minces, parmi lesquelles le YAG dopé $\mathrm{Eu}^{3+} \mathrm{ou} \mathrm{Tb}^{3+}$, pouvant être excitées efficacement par des sources énergétiques même lorsque le matériau est encore amorphe. 
Le YAG en plus de présenter une grande stabilité chimique et mécanique est connu pour être générateur d'une intense fluorescence lorsqu'il est excité par un rayonnement VUV. Les composés YAG dopés $\mathrm{Ce}^{3+}$ ou YAGaG( $\left(\mathrm{Y}_{3}(\mathrm{Al}, \mathrm{Ga})_{5} \mathrm{O}_{12}\right): \mathrm{Tb}^{3+}, \mathrm{Ce}^{3+}$ sont à $\mathrm{l}^{\prime}$ heure actuelle utilisés dans les dispositifs d'éclairage basés sur le pompage optique par diodes bleues. Ce sont des compositions que nous avons élaborées récemment sous forme de poudres et films sol-gel. Les figures 6 et 7 montrent à la fois la qualité optique des couches sol-gel dans le cas du YAG : $\mathrm{Tb}^{3+}$ et le fort recouvrement entre $\mathrm{l}^{\prime}$ émission d'une diode bleue conventionnelle et l'absorption $\mathrm{d}^{\prime}$ une poudre de YAG : $\mathrm{Ce}^{3+}$. Cependant,

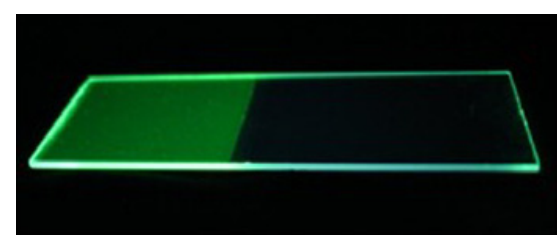

Figure 5. Couche fluorescente de YAG : $20 \% \mathrm{~Tb}^{3+}$ éclairée sous UV.

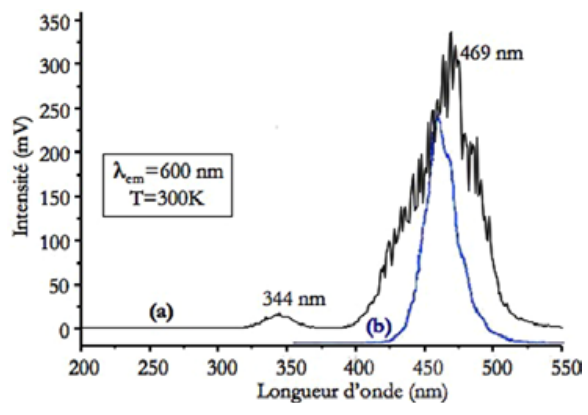

Figure 6. (a) Spectre d'excitation de la fluorescence de $\mathrm{Ce}^{3+}$ dans une poudre cristallisée de YAG:Ce(1\%) comparé au (b) signal d'émission d'une DEL bleue classique à base de GaN .

des efforts restent à faire concernant la structuration de la surface des films (pour atteindre de forts taux d'émissivité perpendiculairement à la surface) et l'élimination de la directionalité des diodes blanches tout en améliorant leur colorimétrie, notamment dans le domaine du rouge. Cette structuration pourra être obtenue en élaborant des couches à structures colonnaires ou en inhibant les effets de guidage et diffusion en augmentant l'épaisseur des couches (sol précurseurs chargés de nanoparticules de même composition ou sol composites silice-nanoparticules). Des développements se font actuellement autour $\mathrm{d}^{\prime}$ autres formulations comme le YAP $\left(\mathrm{YAlO}_{3}\right)$ ou le YAM $\left(\mathrm{Y}_{4} \mathrm{Al}_{2} \mathrm{O}_{9}\right)$ et les polyphosphates $\mathrm{Y}\left(\mathrm{PO}_{3}\right)_{3}$. Ces derniers, qui font partie de la famille des phosphates à anions condensés, sont connus pour présenter une très faible extinction par concentration et ont de réelles potentialités d'application dans le domaine des luminophores pour l'éclairage.

\subsection{Vers le développement de scintillateurs nanostructurés et la contribution à l'élaboration de dispositifs pour l'imagerie médicale}

Bien qu'une recherche intense soit consacrée à la réalisation de détecteurs de rayonnements X et gamma à base de semi-conducteurs II-VI, le développement de détecteurs plans à conversion indirecte associant un scintillateur et un photomultiplicateur ou une caméra CCD reste d'actualité. Les limitations du champ d'applications, comme la moindre résolution spatiale ou le rapport signal sur bruit parfois insuffisant peuvent être fortement améliorés en contrôlant l'architecture et la composition chimique du support. Des avancées importantes ont été réalisées dans l'élaboration de matériaux scintillateurs inorganiques performants par voie sol-gel. Comme nous l'avons signalé, plusieurs familles de matériaux, par ailleurs reconnus comme d'excellent scintillateurs, ont été préparés de façon originale au laboratoire $\left(\mathrm{LnPO}_{4}\right.$, $\mathrm{LnBO}_{3}, \mathrm{Ln}_{2} \mathrm{SiO}_{5}, \mathrm{LnAG}, \mathrm{LiLnF}_{4}, \mathrm{Ln}=\mathrm{Y}, \mathrm{Lu}, \mathrm{Gd}$ dopés par des ions de terres rares) notamment sous forme de couches minces (configuration idéale pour réaliser des écrans). A titre d'exemple, la figure 8 représente un écran sol-gel à base de $\mathrm{LuBO}_{3}: \mathrm{Eu}^{3+}$. Les films sont bien homogènes et une fluorescence est clairement obtenue aussi bien en réflexion qu'en transmission [13]. Cependant, les doses employées pour induire cette fluorescence restent pour l'instant supérieures aux limites admissibles en 
milieu hospitalier. Comme pour les sources d'éclairage, l'accroissement des épaisseurs, tout en gardant la nanostructuration des films nous semble être la solution à même de remédier cet aléa.
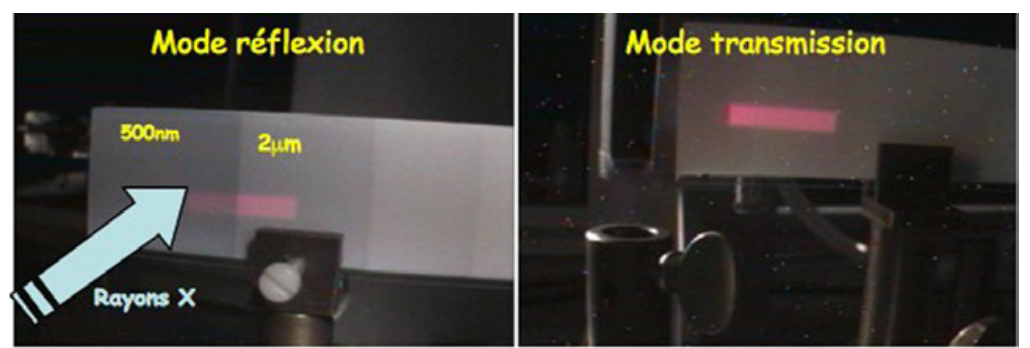

Figure 7. Images de la scintillation en réflexion et en transmission de films spray de diverses épaisseurs de $\mathrm{LuBO}_{3}: 5 \% \mathrm{Eu}^{3+}$.

\section{CONCLUSION}

La technique sol-gel a été utilisée pour élaborer et mettre en forme efficacement de nombreux matériaux luminescents potentiellement utilisables comme luminophores ou scintillateurs. L'accès aux grands instruments et aux lignes de lumière dédiées a permis, en appréhendant l'ordre local, une connaissance plus approfondie de l'état sol. L'étude menée par exemple sur la matrice YAG, a permis d'identifier quelques aspects fondamentaux à prendre en compte lors de l'élaboration et de la conservation des sols. Ils sont dorénavant pris en compte lors de la préparation de nos matériaux et ce quelle que soit leur formulation. Enfin les sources VUV ont été utilisées pour mesurer les réponses spectrales et les rendements lumineux associés en vue des applications visées.

\section{Références}

[1] C.J. Brinker, G.W. Scherer, The Physics and Chemistry of sol gel processing (Academic Press, Boston, USA, 1990)

[2] D.C. Bradley, R. C. Mehrotra, D.P. Gaur, Metal Alkoxides (Academic Press, London UK, 1978).

[3] C. Sanchez, F. Ribot, New J. Chem. 18, (1994) 1007-1047.

[4] D. Boyer, G. Bertrand, R. Mahiou, J. Lumin. 104, (2003) 229-237.

[5] D. Boyer, G. Bertrand-Chadeyron, R. Mahiou, A. Brioude, J. Mugnier, Opt. Mater 24, (2003) $35-41$.

[6] A. Potdevin, G. Chadeyron, D. Boyer, B. Caillier, R. Mahiou, J. Phys. D: Appl. Phys. 38, (2005) 3251-3260.

[7] S. Briche, D. Zambon, D. Boyer, G. Chadeyron, R. Mahiou, Opt. Mater. 28, (2006) 615-620.

[8] E. Pinel, P. Boutinaud, G. Bertrand, C. Caperaa, J. Cellier, R. Mahiou, J. Alloys Compd. 374, (2004) 202-206.

[9] C. Mansuy, R. Mahiou, J.M. Nedelec, Chem. Mat. 15, (2003) 3242-3244.

[10] D. Boyer, R. Mahiou, Chem. Mat. 16, (2004) 2518-2521.

[11] Q.G. Meng, P. Boutinaud, A.C. Franville, H.J. Zhang, R. Mahiou, Microporous Mesoporous Mater. 65, (2003) 127-136.

[12] D. Rodic, M. Mitric, R. Tellgren, H. Rundlof, J. Magn. Magn Mater. 232, (2001) 1-8.

[13] C. Mansuy, G. Chadeyron, J.M. Nedelec, R. Mahiou, C. Dujardin, J. Mugnier, C. Leluyer, B. Peuchot, A. Tanguy, ITBM-RBM 25, (2004) 246-249. 